\title{
Our "Flying Circus" of CIM
}

\author{
M.M.“Mike” Asoodeh, Carl W. Steidley \\ Department of Industrial Technology/Department of Computer Science \\ Southeastern Louisiana University \\ Hammond, Louisiana 70402
}

\section{INTRODUCTION}

We have initiated an affiliation between the Departments of Industrial Technology and Computer Science at Southeastern Louisiana University. This collaboration is part of an on-going effort to develop integrated, undergraduate, technology-rich curricula. We are in the process of creating a model computer integrated manufacturing education facility, using existing equipment and facilities as a basis upon which to build. This facility will be used in courses that emphasize computer-aided manufacturing, computer-controlled systems, robotics, and real-time physical systems with the goal of incorporating the concepts of teamwork, communication, concurrent engineering, and continuous improvement as a major part of the curriculum as well as developing a steady supply of students interested in science, engineering, and technology.

As part of our effort, we are incorporating an outreach component to encourage local students to study science and technology and to learn about the career potentials in these fields. We believe that modern computer integrated technology needs to be incorporated into the school

environment more effectively so that future engineering and science students can acquire interest and skills necessary for their future academic and professional pursuits. Studies have pointed out the inadequacy of typical methods utilized in delivering instruction related to science and technology. The use of hands-on practices has been shown to be an effective tool in delivering instructional materials. These types of activities can aid the students in remembering verbal information and concrete concepts.[1]

We have acquired a mobile mini-CIM cell to be used in our outreach efforts. The portability of this cell allows us to pack up, transport to sites for our "show", in much the same way that the circuses of yesteryear, moved their "big top" tents from site to site to put on their show. This mobile system integrates computer hardware/software, instrumentation, interfacing, computercontrolled systems, real-time systems, and integrated manufacturing.

\section{TEAMWORK}

Circuses depend upon teamwork, and forming teams of people from different technical and specialty fields is an excellent way to respond to any customer's needs. A team may be formed to design a product, produce a product, or accomplish a defined project. One purpose for forming a team is to improve the communication between people and minimize the chance of errors. However, the team can only be successful if members of the team have adequate communication skills.

Project oriented teams are probably the most common type found in manufacturing companies. 
Teams are formed to accomplish a wide range of projects. For example, an intercompany team may be formed to determine the requirements for a new manufacturing facility. It may take six months to a year to complete a project like this.[2]

In a similar manner, a designer, a manufacturing engineer and a quality control specialist may form a team to resolve a specific product failure. This may involve a review of the design calculations, manufacturing processes, and in-process inspection procedures. The team may be together for a few days, a week or a month. In any case, the project team is disbanded upon completion of the specific project.

More and more companies are realizing the advantages of the team approach. An example of this is the recently introduced Saturn automobile. In the early 1980's General Motors decided to design a new automobile from the ground up. To accomplish this, they formed a team of product specialists from various General Motors divisions. Rather than assigning this team to one of the existing GM divisions they chose to form a new company, Saturn. Many companies do not go to the extreme of forming a new company, but they are bringing together technical specialists to effect new product designs.[3]

Teamwork is also key to the successful operation of manufacturing cells on the shop floor. In addition to communication skills, team members need to have excellent technical skills, permitting them to cross-train in tasks performed by other members of the team. This crosstraining allows team members to alternate tasks during a work shift in order to minimize boredom and improve productivity.

We are developing a laboratory that will support four courses (2 Computer Science and 2 Industrial Technology) that emphasize computer hardware, computer-controlled systems, and real-time physical systems. It is planned that industrial technology and computer science courses will be taught collaboratively. It is our plan to involve industrial technology students (IT students) and computer science students (CS students) in team projects in the laboratory. Moreover, we plan that CS students will serve as the system's programmers as well as mentor IT students in the fundamentals of the system's programming languages. Likewise, we plan that IT students will serve as manufacturing systems designers as well as mentor CS students in the fundamentals of computer aided design and industrial CNC machinery and its uses in manufacturing processes.

\section{Concurrent Engineering}

"Concurrent engineering" is a specific team approach that is being used to improve product design and to decrease the time required to bring the product to the marketplace. A relatively new concept, concurrent engineering is a systematic approach to the simultaneous design of products and their related processes. All elements of the product life cycle, from concept to disposal, are considered in this approach.

It seems that this logical strategy for the design and manufacture of a product should already be in place in most companies. If not in place, it seems likely that companies would want to 
implement concurrent engineering. Unfortunately, most U.S. companies have been slow to change to this new concept of product development and design. They resist the change because of existing management structures.

The typical company is organized along functional lines. This includes sales, engineering, manufacturing, assembly and customer service. Each of these groups have their own management structure and tended to operate autonomously. Such a structure results in the sequential engineering of a product. Each activity is completed by one group, which then passes it to the next group. Once a group has performed its assigned tasks, the product is moved to each successive group until it is shipped.

The sequential design process is usually done with very little communication between groups. It is as if walls are placed between the functional groups, hindering direct communication. In a sense, the design project moves through the organization by passing over the "wall" to the next group in the series. There is also a greater risk that the product will not satisfy the customer's requirements as a result of the lack of communication between the functional groups during the design process.

The concurrent engineering team approach results in a two-fold benefit. First, the product is designed to satisfy the needs of the customer, including price, delivery and quality. The second benefit of this approach is that the manufacturing processes necessary to produce the product are designed along with the product. On-going communication between team members is necessary to assure that a product will meet the requirements of the customer.[4]

In our "flying circus" of CIM, students will not only gain hands-on experience with the fundamental hardware building blocks of automated systems, but will integrate these building blocks with real-time software systems to develop collaborative, interdisciplinary, real-world, computer-controlled manufacturing systems with which they will produce a finished product in capstone fashion. In this manner we expect that our students will be forced to practice concurrent engineering.

\section{Continuous Improvement}

Another fundamental concept of modern product management is "continuous improvement." Continuous improvement is a process involving all employees. It virtually empowers all employees to strive to improve the activities in which they are involved. The goal of continuous improvement is to provide the customer with a product which continues to improve over time.

The basic premise of continuous improvement is simple: Environments and conditions change over time. These changes bring about the need for improvements in processes which improve quality in a product or service. As new information becomes available, opportunities for improvement (better, faster, cheaper ways of doing things) present themselves. Continuous improvement is an ongoing, never ending process, not a one time project with a beginning and end. It is a philosophy of business as well as personal life. 
Implementation of the continuous improvement process, along with other aspects of CIM, is very difficult, especially with our current manufacturing culture. Most of our manufacturing management principles were conceived in the early 1900's. The success of assembly line mass production methods, started by Henry Ford, has served the manufacturing community well for many years. The premise of this older manufacturing method was that each employee had only to do his or her job with very little thought or training. The manufacturing and assembly tasks were broken down into a series of very simple actions. Although this permitted maximum production output it did not always foster a quality product.

When tasks are broken down to almost meaningless actions that require little thought by the employee, the employee takes little "ownership" in the overall product. The employee was not expected to report things that were wrong or even assure that his own job is done correctly every time. The overall goal was to mass produce. Quality was not a major concern. After all, business in the U.S. had a captive domestic market and little, if any, foreign competition when this management method was developed.

With worldwide competition and the implementation of increasingly sophisticated equipment and systems, business has recognized the need to have a trained workforce of thinking employees. Business has acknowledged that employees are the most valuable asset. If the U.S. is to be a successful competitor in the worldwide marketplace, it is essential that an educated workforce know, understand, and apply the principles of continuous improvement.[5]

We believe that our approach will provide our students with a more complete exposure to the intricacies of proper "continuous improvement." This approach will allow us to cut across the traditional disciplinary boundaries. These courses will emphasize the use of the computer in design and development of factory automation. We believe that the efforts of this project will provide our students with an improved ability to express ideas quickly and accurately.

\section{Use of Computer Modeling Technologies}

We are making computer simulation and modeling an integral part of our project. Rapid advances in computer technology coupled with the advancement in computer graphics software make the task of combining visualization and design more feasible.

In our laboratory, functions of modeling, analysis and visualization are merged. For us modeling refers to geometric and non-geometric product data and is usually performed by computer aided design (CAD) systems. The analysis is the usual task of computer aided engineering (CAE) systems and components. The product shape, characteristics, and results of analysis may be performed by the visualization module or system. Any of the three components may be displayed at any time during the design process. 


\section{Outreach Activities}

Students at all academic levels need outreach and enrichment programs that bring them into the mathematics, science, and engineering career pipeline. In addition, they need general education and the ancillary skills necessary for success in mathematics and science. Thus, educational programs need to be broadly based efforts that actively involve K-12 teachers, college and university faculty, industry, community organizations, and parents in outreach and enrichment activities. These efforts should include a focus on the development of each group's capacity to offer such programs.[6]

Outreach and enrichment activities must complement and enhance instructional programs in schools, colleges, universities, museums, and other educational institutions, and they must evolve with these programs as reforms in mathematics and science education occur. We believe that increased representation in mathematics, science, and engineering careers depends on a careful articulation of outreach efforts for students, faculty, and the community that provide immediate enrichment and information for students and their parents.

The "flying circus" CIM cell system described later in this paper may be also used for seminars, workshops and public demos to introduce the local school audience to manufacturing technology, scientific concepts, multimedia, Internet, WWW, and other advanced technologies. It is our intent that with this equipment students at local schools will gain hands-on experience with these technologies. Our challenge and need is to teach these students how to think and solve problems using computer systems before they come to our university.

We are developing a series of training exercises and demonstrations in the design and use of CIM. This process will also include the development of on-line demo modules. These modules will serve to raise the sophistication level of the users according to the users needs. Much like "on-demand" manufacturing processes where products are produced as needed, demos will be presented as needed by the user. One of the essential components of a truly effective network is the bringing together of university and local schools for a common purpose. Partnerships are an integral part of this collaboration and one of the many strengths that may be drawn on in this project. We are in the process of establishing partnerships, clarifying the roles and responsibilities of the partners, as well as seeking new partners.

Our students will contribute to the development of demos and mentoring local schools' students in using them. They will also serve as assistants, help session/recitation leaders, and graders to our own students for activities that they have already completed. Students at all levels will be selected to serve on the development teams. However, these basic principals will be implemented: first, all selections will be a special recognition in which mastery of the content and dedication will be a primary factor, secondly, this privilege will open additional opportunities for participation in the educational process while committing themselves to a larger amount of responsibility. These opportunities will help students to accept responsibility for a part of their own education and to see themselves as active learners rather than that of passive recipients with continuous lecture. 


\section{THE ROAD SHOW}

The AMS-107 Mini CIM Cell is a complete mobile computer-integrated manufacturing system consisting of robot, parts feeder, programmable logic controller (PLC), sensor package, CNC milling machine, and system software, which allows us to teach the fundamentals aspects of industrial automation, robotics, controls, CNC, sensors, systems integration, and computer-aided manufacturing. The system consists of industrial grade components that can function together to manufacture parts automatically in a laboratory or demonstration setting. It is designed so that each subsystem of the total system can be used as a stand-alone teaching tool and the entire system is contained on an anodized extruded tubing frame mounted on casters with emergency stops and a table top that is 78" x 24 " standing 36 3/4" high.

The cell's robot is TII's B901 four-axis, five-degree-of-freedom pneumatic robot. This robot is modular by design allowing for interfacing with various pneumatic training systems, RS232 computer interface, and/or PLCs. It is equipped with a hand-held teaching pendant for manual operation and control. The manipulator or gripper is industrial-grade and is equipped with removable fingers for greater end-of-arm tooling flexibility. The arm is capable of a full 180 degrees of motion and is equipped with an air over oil control system that allows for smooth speed control of up to 180 degrees per second. The arm can extend 3 inches in the horizontal plane and 4 inches in the vertical plane and is capable of carrying a full arm extension payload of 11 pounds with a deflection of only .0005 inches. Arm rotation and linear motion can be adjusted using mechanical stops. The wrist is capable of 180 degree rotation with speeds up to 180 degrees per one and one-half second.

The parts feeder is a magazine-type pneumatic parts feeder. This feeder is specifically designed to dispense flat approximately 5 inch by 3 inch by $1 / 2$ inch parts. It is equipped with an optical sensor to determine the presence or absence of parts.

The cell is equipped with an Allen Bradley SLC-100 PLC. The PLC can be programmed by the hand-held pendant, EEPROM, or by online/offline software programming on a PC and downloading via an RS232 interface converter.

The cell includes a suite of industrial grade sensors used for controlling the system. The system's sensor suite includes: roller lever limit switches, standard lever limit switches, diffusereflective fiber optic cable, thru-beam fiber optic cable, infrared devices, and proximity devices.

The Mini Mill is a durable PC controlled CNC milling machine specifically designed for desktop manufacturing applications. The system uses standard CNC G\&M codes that conform to the EIA RS-274D standard NC machine language. The mill has an $\mathrm{x}$-axis travel capability of 10 inches, $y$-axis travel of 5 inches, and z-axis travel of 7 inches. The machine is capable of simultaneous three-axis helical, circular, and linear interpolation. It is equipped with pneumatic vise and is capable of milling everything from wax to steel.

The system software consists of online/offline software for controlling the PLC, CNC milling machine software, and CAD software. 
The PLC software package offers complete programming/documentation and reporting, as well as upload/download capabilities. The software offers a number of user-friendly features including: a full-screen rung display for PLC ladder logic programs, symbolic and/or address programming, in-line documentation features such as; rung description, instruction description, and sequencer programming tables. The software also provides a number of useful reports. For example, full cross-reference and usage reports for timers, counters, and bits. This is an interactive application package which assists the user through designing, coding editing, filing, and ultimately machining a CNC part. The software is menu driven with EIA standard RS-274 D CNC format. It provides for simultaneous 3-axis helical, circular and linear interpolation with the capability of having X,Y,Z -I,J,K coordinates. Additional capabilities include: full screen CNC code generation/editing with verification graphic tool path simulation and verification prior to machining; G\&M code look-up tables with definition and usage; single or continuous block milling and simulation; inch and metric programming; and mixed incremental/absolute programming.

This software converts CAD drawings into CNC code without the need for any CAD, CAM, or CNC training. General features of the package include: absolute and incremental programming; program memory of 6,500 blocks per part program; creates both job and block file (machine type and program); menu driven.

The software that we are using with this system for CAD design and development is TurboCAD. General features include: high precision drawing; 256 layers; selectable line and arrow types; multiple set fonts; automatic and associative dimensioning; draw any geometric shape; multiple drawing windows; easy symbol and group selection; time saving macro language; print and plot spooling; bill of materials capability. The editing features include: linear, radial, or array copying; 18 snap modes; scale, rotate or move; unlimited zoom and pan choice of pen and screen colors. Minimum printing features must be all common printers and plotters; no additional hardware required; import and export in DXF, HPGL, or ASCII formats to other software programs.

\section{Conclusions}

Pre-college students should interact regularly with undergraduate and graduate students. Students at all academic levels should interact with scientists and engineers in a variety of learning situations. Beginning at the pre-college and continuing through all academic levels, students must be aware of career requirements, undergraduate opportunities, and graduate school options. As part of these efforts, "Near Peer" mentoring can play an important role - high school students mentoring middle school students, middle school students mentoring at the elementary level.

We are developing a state of the art model integrated facility that is used to impart software and hardware principles to future scientists, engineers, managers, designers, and technical supervisors. Our challenge and need is to teach these students how to think and solve problems in terms of automated systems. We believe this goal is better accomplished through close ties with our community that will utilize this knowledge base. The activities listed in this paper will 
infuse integrated technologies and their uses into the classroom. These technologies will be representative of the types of technology that students will face in the workforce.

\section{References}

1. Bell, T., \& Elmquist, 1992, Technical Interaction in the Classroom. Vocational Education Journal, 67(3), pp. 22-24.

2. Abernathy, W.J., K.B. Clark, and A.M. Kanthrow, Industrial Renaissance: Producing a Competitive Future for America, New York, Basic Books, 1983.

3. Cohen, S.S., and J. Zysman, The Myth of the Post-Industrial Economy, Siemens Review, 55, March/April, 1988.

4. Suri, R, and S. DeTreville, Getting from 'Just-in-Case' to 'Just-in-Time': Insights from a Simple Model, Journal of Operations management, 295, May, 1986.

5. National Academy of Sciences, Balancing the National Interest: U.S. National Security Export Controls and Global Economic Competition, Washington, D.C., National Academy Press, 1987.

6. Quality Education for Minorities in Mathematics, Science, and Engineering Network, Together We Can Make It Work, Washington, D.C., 1992.

\section{Biographical Information}

MOHAMMED M. "MIKE" ASOODEH is an Assistant Professor of Industrial Technology. He earned his Ph.D. at Texas A\&M University. His research interests are in telecommunications, systems engineering, and robotics with current emphasis on multimedia development to support his teaching in these areas.

CARL STEIDLEY is a Professor of Computer Science. He earned his Ph.D. at the University of Oregon. His interests are in the applications of artificial intelligence and robotics. Prior to joining the faculty at Southeastern La. Univ. he taught computer science at Central Washington Univ., and Austin Peay State Univ. Before that he was a member of the faculty of mathematics and physics at Oregon Institute of Technology. He has recent research and development appointments at NASA Ames Research Center and Oak Ridge Natl. Labs 